This is the final peer-reviewed accepted manuscript of:

Chen, J., Valsecchi, M., \& Gegenfurtner, K. R. (2017). Attention is allocated closely ahead of the target during smooth pursuit eye movements: Evidence from EEG frequency tagging. Neuropsychologia, 102, 206-216. doi:

https://doi.org/10.1016/j.neuropsychologia.2017.06.024

The final published version is available online at:

https://doi.org/10.1016/i.neuropsychologia.2017.06.024

Rights / License:

The terms and conditions for the reuse of this version of the manuscript are specified in the publishing policy. For all terms of use and more information see the publisher's website. 


\title{
Attention is allocated closely ahead of the target during smooth pursuit eye movements: Evidence from EEG frequency tagging
}

\author{
Jing Chen, Matteo Valsecchi, Karl R. Gegenfurtner* \\ Abteilung Allgemeine Psychologie, Justus-Liebig-Universität Gießen, 35394 Gießen, Germany
}

\begin{abstract}
A B S T R A C T
It is under debate whether attention during smooth pursuit is centered right on the pursuit target or allocated preferentially ahead of it. Attentional deployment was previously probed using a secondary task, which might have altered attention allocation and led to inconsistent findings. We measured frequency-tagged steady-state visual evoked potentials (SSVEP) to measure attention allocation in the absence of any secondary probing task. The observers pursued a moving dot while stimuli flickering at different frequencies were presented at various locations ahead or behind the pursuit target. We observed a significant increase in EEG power at the flicker frequency of the stimulus in front of the pursuit target, compared to the frequency of the stimulus behind. When testing many different locations, we found that the enhancement was detectable up to about $1.5^{\circ}$ ahead during pursuit, but vanished by $3.5^{\circ}$. In a control condition using attentional cueing during fixation, we did observe an enhanced EEG response to stimuli at this eccentricity, indicating that the focus of attention during pursuit is narrower than allowed for by the resolution of the attentional system. In a third experiment, we ruled out the possibility that the SSVEP enhancement was a byproduct of the catch-up saccades occurring during pursuit. Overall, we showed that attention is on average allocated ahead of the pursuit target during smooth pursuit. EEG frequency tagging seems to be a powerful technique that allows for the investigation of attention/perception implicitly when an overt task would be confounding.

Keywords:

Attention

Smooth pursuit eye movements

Frequency tagging

SSVEP
\end{abstract}

\section{Introduction}

Moving objects in the environment are among the most salient inputs to our visual system (Dorr et al., 2010; Itti, 2005). Smooth pursuit eye movements have the function of stabilizing moving objects on the retina and keeping them in the fovea, enabling high-resolution visual analysis. Eye movements are tightly linked to spatial attention in the visual system (Deubel and Schneider, 1996; Kowler et al., 1995; Hoffman and Subramaniam, 1995; Rizzolatti et al., 1987; Awh et al., 2006; Smith and Schenk, 2012). Studies of eye movements and attention, however, have almost exclusively focused on saccades (Corbetta et al., 1998; Moore and Armstrong, 2003; McPeek and Keller, 2004; Carello and Krauzlis, 2004; Müller et al., 2005; Belopolsky and Theeuwes, 2012; MacLean et al., 2015) and microsaccades (Engbert and Kliegl, 2003; Laubrock et al., 2007; Turatto et al., 2007; Hafed et al., 2011; Yuval-Greenberg et al., 2014; Chen et al., 2015). The few studies that investigated the relationship between visual attention allocation and smooth pursuit eye movements in the last decades (Lovejoy et al., 2009; Khan et al., 2010; Seya and Mori, 2012;
Watamaniuk and Heinen, 2015) gave conflicting answers to the question whether attention is centered on the target of pursuit or allocated preferentially ahead of it.

These previous studies used dual-task paradigms with a secondary attention-probing task on top of the smooth pursuit task. The secondary task varied from a saccadic reaction time task (Tanaka et al., 1998; Kanai et al., 2003; Khan et al., 2010), to a manual button pressing reaction time task (van Donkelaar, 1999; van Donkelaar and Drew, 2002; Khan et al., 2010; Seya and Mori, 2012), to a perceptual discrimination task (Lovejoy et al., 2009; Harrison et al., 2014; Watamaniuk and Heinen, 2015). Reaction times or discrimination accuracy were used to infer the amount of attention at various locations relative to the target of pursuit. Some studies reported that attention was symmetrically allocated around the target location (e.g., Lovejoy et al., 2009; Watamaniuk and Heinen, 2015), while others found that it was focused slightly ahead of the target (e.g., van Donkelaar and Drew, 2002), or broadly ahead of it (e.g., Khan et al., 2010).

The use of a secondary attention-probing task during smooth pursuit can be problematic. It is well known that the control of attention is 
flexible, and highly dependent on the task requirements (Müller and Hübner, 2002; Müller et al., 2003; McMains and Somers, 2004, 2005). Given that smooth pursuit itself is not a demanding task (Hutton and Tegally, 2005; Seya and Mori, 2015), the allocation of attention likely had adapted to the demands of the secondary task in previous studies. The default bias induced by pursuit, if it exists, might have survived (as suggested in Khan et al., 2010) or have been overridden (as discussed in Watamaniuk and Heinen, 2015).

To circumvent the problems produced by the attention-probing task, we adopt an implicit measurement of attention - the frequency tagging technique based on steady state visual evoked potentials (SSVEP). SSVEP is an oscillatory brain response to periodic visual stimulations (see Norcia et al., 2015 for review). SSVEP amplitude increases with more attention (Morgan et al., 1996), and has been used widely as an index of attention allocation (see Andersen et al., 2011 for review). In the present study, patterns flickering at different frequencies were displayed at different locations relative to the target of pursuit. Frequency-tagged SSVEPs were recorded to concurrently access the allocation of attention to these locations. We specifically aim to find out whether SSVEP induced by stimuli ahead of the stimulus in the direction of motion is enhanced and, if so, how far ahead the enhancement extends. Our results suggest that attention is allocated closely in front of the pursuit target (Experiment 1) but not extended far ahead of it (Experiment 2), and that the effect is not driven by catch-up saccades (Experiment 3).

\section{Materials and methods}

Three experiments were conducted in agreement with the Declaration of Helsinki and following guidelines approved by the local ethics committee (2013-0018). All observers signed informed consent forms before taking part in the experiment, and were naïve to the purpose of the study at the time of testing. They had normal or corrected-to-normal vision and had no known neurological or oculomotor diseases.

\subsection{Apparatus}

Stimuli were displayed using the Psychophysics Toolbox (Brainard, 1997; Pelli, 1997) in MATLAB (MathWorks, Natick, MA, USA), on a $120 \mathrm{~Hz}$ Samsung SyncMaster 2230R7 22-in. monitor (Samsung Group, Seoul, South Korea). With a spatial resolution of $1680 \times 1050$ pixels, the screen extended $61^{\circ}$ horizontally and $38^{\circ}$ vertically at a viewing distance of $40 \mathrm{~cm}$. Stimuli were presented on a gray background $\left(111.6 \mathrm{~cd} / \mathrm{m}^{2}\right)$.

\subsection{Experiment 1: Two locations}

We frequency-tagged two locations, in front and behind the pursuit target, in order to examine whether attention is enhanced in front of the pursuit target.

\subsubsection{Participants}

Twelve observers ( 5 females and 7 males, age 19-45, average 27 years) participated in this experiment.

\subsubsection{Stimulus}

The target for smooth pursuit was a black bull's eye, with $0.07^{\circ}$ inner radius and $0.29^{\circ}$ outer radius (Fig. 1A). The flicker stimuli were black and white checkerboard patterns located on the left and right side of the pursuit target. Each element in the patterns had a size of $0.73^{\circ} \times$ $0.73^{\circ}$. The stimulus extended $8.15^{\circ}$ horizontally and $8.15^{\circ}$ vertically, and was filtered through a $2 \mathrm{D}$ Gaussian window $\left(\mathrm{SD}=1.46^{\circ}\right.$ ). The left and right checkerboards were separated by a $0.15^{\circ}$ gap to prevent interactions at the border. The left and right stimuli were reversing polarity every 18 frames or every 16 frames, which resulted in a pattern- reversal frequency of either $6.7 \mathrm{~Hz}$ or $7.5 \mathrm{~Hz}$. We chose to use these two frequencies as 1) they differ enough that we could resolve their individual peak responses in frequency domain (see Fig. 2 and Fig. 5), and 2) their speeds of flickering are close enough that observers get roughly balanced visual experiences on the two sides. We ensured precise timing in the presentation of the flicker by monitoring the flip time after the display of each frame. If occasionally a frame was not presented at the requested time, the next frame was also skipped to make sure that the overall flickering sequence maintained the same phase.

The stimulus moved horizontally within a range of $35.8^{\circ}$ centered on the screen. It took $5 \mathrm{~s}$ for the target to move from one side to the other side. The velocity profile consisted of an increasing phase $(0.83 \mathrm{~s})$, a steady phase $(3.33 \mathrm{~s})$, and a decreasing phase $(0.83 \mathrm{~s})$. In the increasing phase, the velocity grew linearly from $0,2.19,4.39$, $6.58-8.77 \mathrm{deg} / \mathrm{s}$ (corresponding to a target displacement of $0,1,2,3$, or 4 pixels for every two refreshing frames). In the steady phase, the velocity was kept constant at $8.77 \mathrm{deg} / \mathrm{s}$. In the decreasing phase, the velocity decreased linearly from 8.77 to $0 \mathrm{deg} / \mathrm{s}$. We chose to maximize the length of epochs at a constant speed, as the attentional effect during pursuit has been suggested to vary with pursuit speed (Khan et al., 2010). The acceleration and deceleration phase was included to ensure a smooth switch at the turning point. Each pursuit trial lasted $150 \mathrm{~s}$ with 15 cycles of such movements.

\subsubsection{Procedure}

The experiment was conducted in a dimly lit room. In pursuit trials, the target stimulus moved horizontally for $150 \mathrm{~s}$, with the front part flickering at $6.7 \mathrm{~Hz}$ (or $7.5 \mathrm{~Hz}$ ) and the back part flickering at $7.5 \mathrm{~Hz}$ (or $6.7 \mathrm{~Hz}$ ). When the stimulus reversed its direction at every $5 \mathrm{~s}$ mark, the front and back frequencies were also switched, so that one specific flicker frequency (e.g., the $6.7 \mathrm{~Hz}$ flicker) was always assigned to the side ahead of the target in the direction of pursuit within a trial. Each observer completed two pursuit trials in order to balance the two frequencies. Except the first 3 observers, the remaining 9 observers in addition underwent 2 fixation trials. During fixation trials, the stimulus remained stationary in the center, and participants were required to fixate on the central spot. For the first 3 participants, the left flicker was flickering at $6.7 \mathrm{~Hz}$ while the right one was flickering at $7.5 \mathrm{~Hz}$ for one fixation trial, and vice-versa for the other fixation trials. For the remaining 9 participants, the two flickers were presented exactly as they were in the two pursuit trials. Specifically, the two flickers switched positions at every $5 \mathrm{~s}$ mark as if it was in a pursuit trial. The sequence of 4 trials was randomized.

\subsection{Experiment 2: Multiple locations}

We further tested 8 locations around the pursuit target to investigate the spatial selectivity of the attentional enhancement during smooth pursuit.

\subsubsection{Participants}

Twelve observers (10 females and 2 males, age 18-33, average 25 years) took part in this experiment. None of them participated Experiment 1.

\subsubsection{Stimulus}

The stimulus consisted of eight $100 \%$ contrast sectors around the central fixation spot (Fig. 1B). The sectors were centered along the four cardinal directions. Each sector had a central angle of $50^{\circ}$ and 50 pixels in radius $\left(1.8^{\circ}\right.$ in visual angle, from 0.58 to $2.38^{\circ}$ for the inner sectors and from 2.59 to $4.39^{\circ}$ for the outer sectors). The area of the outer sectors was 2.4 times larger than the one of the inner sectors. As SSVEP responses decrease with increasing eccentricity, this design ensured comparable SSVEP responses to sectors located at different eccentricities. Eight sectors flickered at 8 different frequencies, i.e., $5.2 \mathrm{~Hz}$, $6.3 \mathrm{~Hz}, 7.5 \mathrm{~Hz}, 8.6 \mathrm{~Hz}, 10 \mathrm{~Hz}, 10.9 \mathrm{~Hz}, 12 \mathrm{~Hz}$, and $13.3 \mathrm{~Hz}$. They were 
A

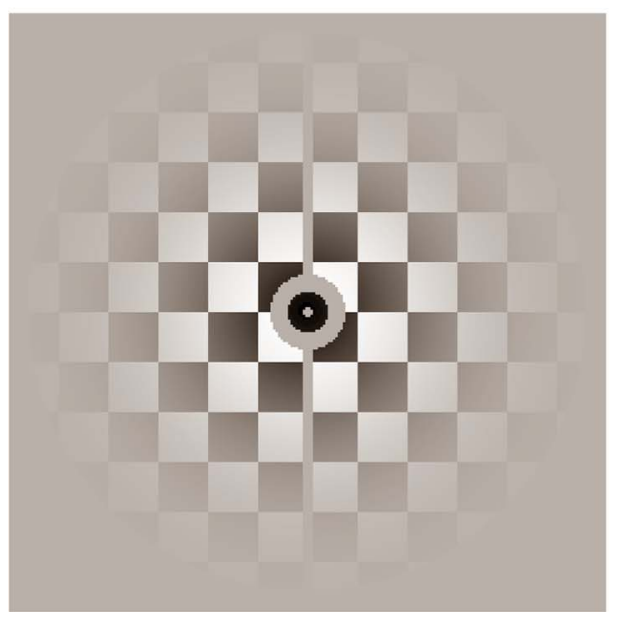

B
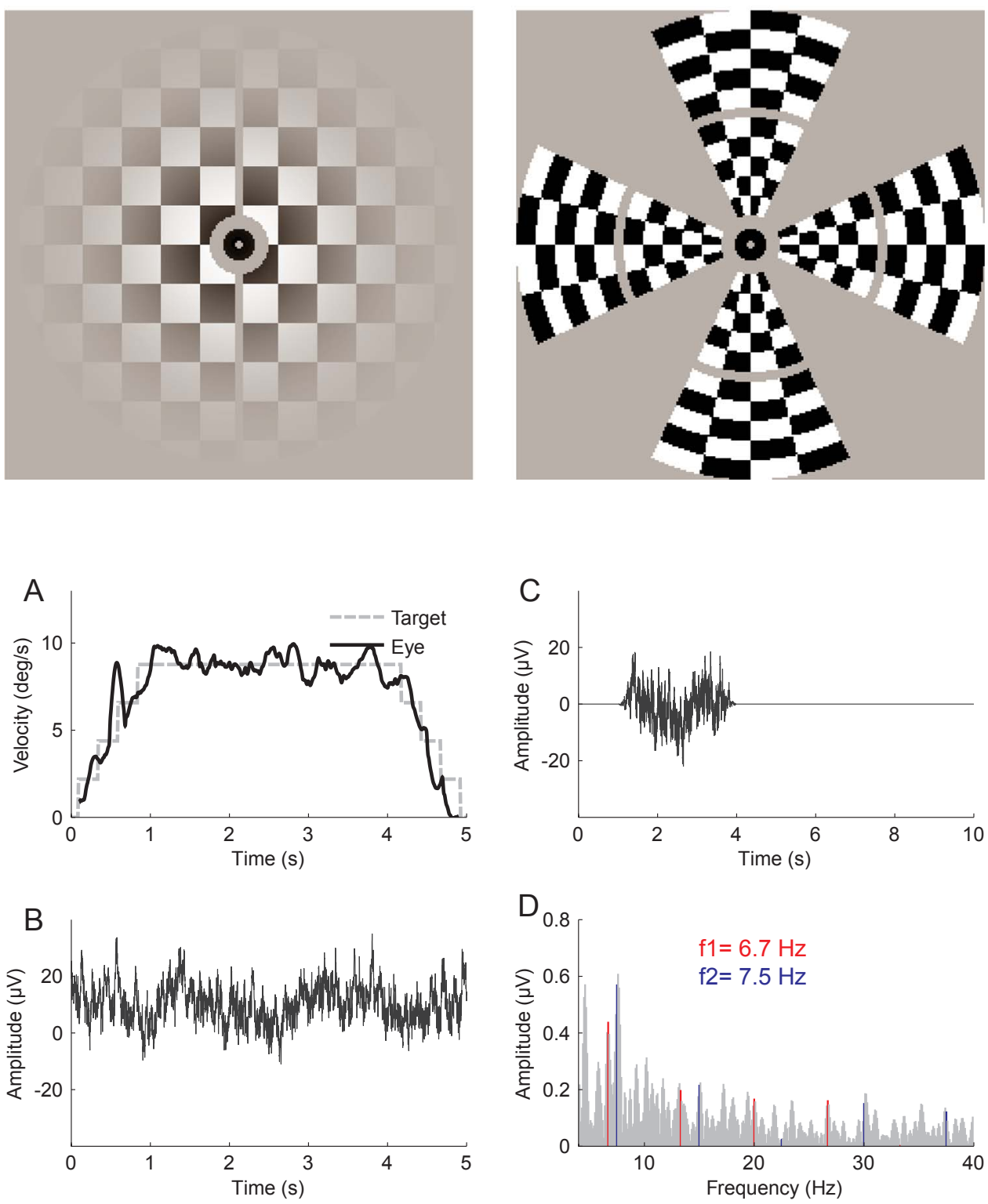

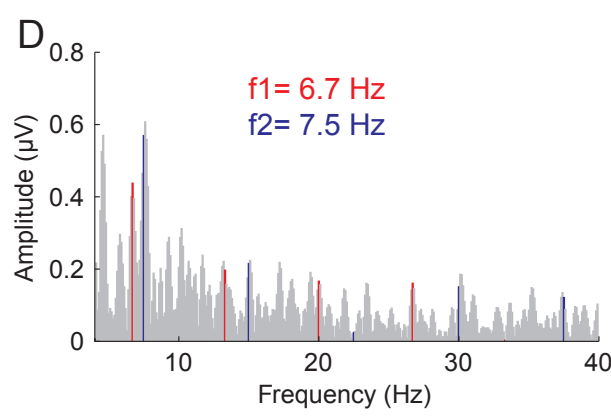

Fig. 1. Displays used in Experiment 1 (A) and Experiment 2 (B). The central black bull's eye served as the target for pursuit or fixation. In smooth pursuit condition, the whole display moves together at the same speed. Checkerboard patterns were counterphase flickering at difference frequencies (A, 6.7 and $7.5 \mathrm{~Hz}$ alternatively on each side; $\mathrm{B}, 5.2,6.3,7.5$, $8.6,10,10.9,12$, and $13.3 \mathrm{~Hz}$ balanced across the 8 sector components). EEG power at these frequencies was measured to infer the amount of attentional resources allocated to the stimulus area. separated by $\sim 1 \mathrm{~Hz}$. None of them overlapped with the other's 1 st or 2nd harmonics (i.e., we did not include $5 \mathrm{~Hz}$, as this would have elicited SSVEP responses at $10 \mathrm{~Hz}$ as well). The arrangement of frequencies at 8 locations was balanced across observers with a Latin square design. Within each observer, the frequency arrangement was balanced along the horizontal and the vertical axis. For example, for Subject 1, the order of frequencies on the horizontal axis was [5.2 6.312 .013 .3$] \mathrm{Hz}$ in half of the trials and was [13.3 12.06 .35 .2$] \mathrm{Hz}$ in the other half. The movement of the stimulus in Experiment 2 was the same as that in Experiment 1.

\subsubsection{Procedure}

Each participant underwent 6 trials including 2 fixation trials, 2 pursuit trials and 2 "attend-while-fixating" trials. Fixation trials and pursuit trials were the same as that in Experiment 1 except for the stimulus display. In Experiment 2, we included 2 "attend-while-fixating" trials, in which the stimulus remained stationary and participants were required to maintain fixation in the center while paying attention to one side of the sector in order to perform a counting task. To mimic the inferred allocation of attention in the pursuit task, we also switched every $5 \mathrm{~s}$ the side participants had to attend (as participants switched pursuit direction every $5 \mathrm{~s}$ in pursuit trials). A small black cue $\left(0.11^{\circ}\right.$ in radius) either to the left side or to the right side of the fixation center indicated which side participants had to attend. During each $150 \mathrm{~s}$ trial, the horizontal outer sectors could disappear for a duration of $92 \mathrm{~ms}$ (i.e., 11 frames) for 5-12 times. The participants' task in the "attendwhile-fixating" trials was to count the number of times the stimulus disappeared on the cued side. Note that the attentional cue and the disappearing of the outer sectors were presented in fixation and pursuit trials as well (irrelevant to the fixation/pursuit task). The visual displays were therefore identical in 3 conditions. We tested the 4 fixation/ pursuit trials (in randomized order) before the 2 "attend-while-fixating" trials. Participants were not instructed on the black cue and the occasional disappearing of sectors until they finished the fixation/pursuit trials. In this way, we aimed at minimizing the potential influence of the task set from the "attend-while-fixating" trials on the fixation/ pursuit trials.

\subsection{Experiment 3: Effects of saccades}

In Experiment 3, we used higher flicker frequencies. Using higher flicker frequencies allowed us to identify the SSVEP signal within 


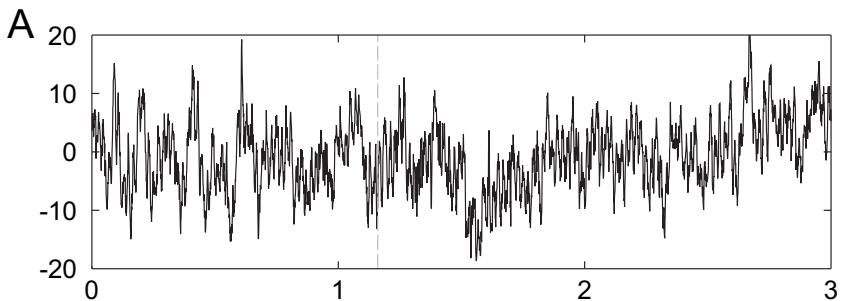

B
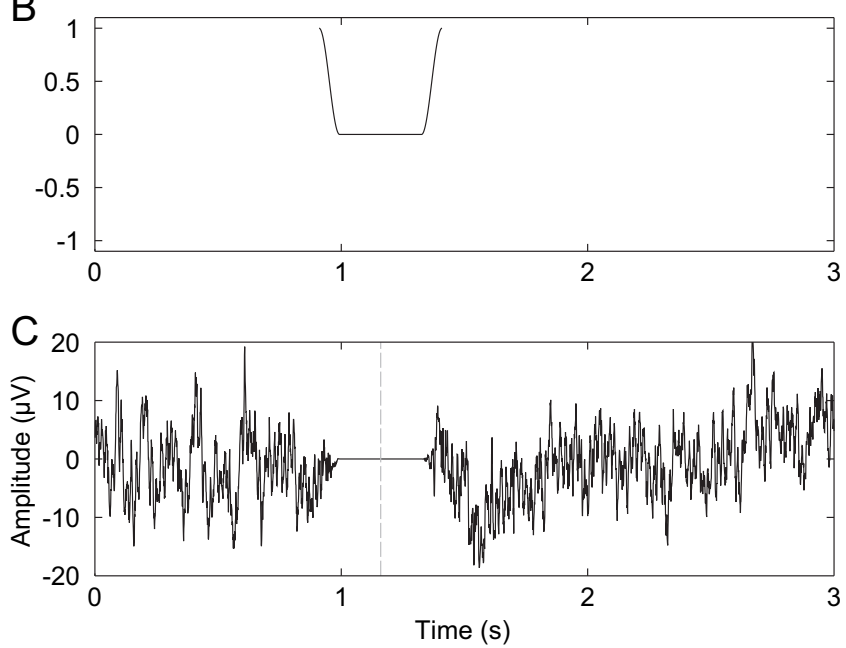

Fig. 3. Procedure used to remove the EEG signals locked to saccades in Experiment 3. A, a sample EEG epoch in which a saccade occurred at $1.16 \mathrm{~s}$ marked by the gray dashed line. $B$, the window function which was multiplied with the EEG epoch, [-250 250] ms locked to saccade onset. C, the resulting epoch, which entered further analysis.

shorter epochs, and to discard recording segments contaminated by catch-up saccades.

\subsubsection{Participants}

Sixteen observers (7 females and 9 males, age 21-47, average 26 years) took part in this experiment. None of them participated Experiment 1 or 2 .

\subsubsection{Stimulus and procedure}

The stimulus was the same as that in Experiment 1. It was presented at a viewing distance of $50 \mathrm{~cm}$. In Experiment 3, we aimed to analyze EEG signals when participants were executing purely smooth pursuit eye movements, after discarding signals when saccades occurred. It would result in shorter duration signals. We thus increased the patternreversal frequencies to $20 \mathrm{~Hz}$ and $24 \mathrm{~Hz}$, which allowed SSVEP analysis on signals with shorter length. In order to reduce the amount of catchup saccades during smooth pursuit, the movement of the stimulus in each trial lasted $5 \mathrm{~s}$, instead of $150 \mathrm{~s}$ in Experiment 1 and 2. The stimulus moved either from the left to the right side, or from the right to the left side. The moving direction and the location of $20 / 24 \mathrm{~Hz}$ flickers were balanced. There were 60 trials in total. The motion speed was constant $(7.3 \mathrm{deg} / \mathrm{s})$.

\subsection{Eye movement recording and analysis}

Eye movements of the right eye were recorded at $1000 \mathrm{~Hz}$ using an Eyelink 1000 table-mounted eye tracker (SR Research, Missisauga, ON, Canada). A chin rest was used to limit the head movements. We define as positive gaze error values the errors where gaze leads the target in the real (Pursuit condition) direction of pursuit, in the simulated direction of pursuit (Fixation condition), or in the direction indicated by the cue (Attend-while-Fixating condition), while negative values indicate that the eye is lagging.

\subsection{EEG recording and analysis}

EEG was recorded from 32 scalp sites according to the international 10-20 system (FP1, FP2, F3, F4, C3, C4, P3, P4, O1, O2, F7, F8, T7, T8, P7, P8, Fz, Pz, Oz, FC1, FC2, CP1, CP2, FC5, FC6, CP5, CP6, TP9, TP10, HLeo, Veo, HReo). Signals were amplified (Brain Products $\mathrm{GmbH}$, Munich, Germany) and sampled at $1000 \mathrm{~Hz}$. The ground electrode was placed at the $\mathrm{AFz}$ location, and the on-line reference electrode at the $\mathrm{Cz}$ location. Electrode impedances were kept below $5 \mathrm{k} \Omega$.

Analyses were carried out with functions from EEGlab toolbox (Delorme and Makeig, 2004) and customized scripts in MatLab. EEG signals were re-referenced to a common average reference. Thirty successive $5 \mathrm{~s}$ epochs were extracted from each $150 \mathrm{~s}$ trial. Signal processing procedure was illustrated in Fig. 2 . We ignored the signal of the first second and the last second in each $5 \mathrm{~s}$ epoch, because they involved speed increase or decrease, respectively, in target motion (Fig. 2A). The remaining $3 \mathrm{~s}$ was first de-trended by removing the linear fit (Bach and Meigen, 1999), and multiplied by a Tukey window (i.e., tapered cosine window, alpha $=0.33$ ). We then zero-padded the signal to $10 \mathrm{~s}$ to get a frequency resolution of $0.1 \mathrm{~Hz}$ (e.g., Gray et al., 2015). Fast Fourier Transformation ( $f f . m$ in MatLab) was used to obtain the amplitude spectrum (Fig. 2D). In Experiment 1, SSVEP amplitude for each flicker (either 6.7 or $7.5 \mathrm{~Hz}$ ) was calculated by averaging 5 harmonics weighted by their signal-noise-ratio (SNR), so that the harmonic with higher SNR contributes more to the combined SSVEP amplitude, with the following formula (Zhang et al., 2011): $\mathrm{A}_{\text {combine }}=\left(\frac{\sum_{i=1}^{5} A_{i}^{2} \times S N R_{i}}{\sum_{i=1}^{5} S N R_{i}}\right)^{1 / 2}$. Amplitude at the $i^{\text {th }}$ harmonic $\left(A_{i}\right)$ was computed as the mean of the 3 bins around the harmonic (e.g., for $7.5 \mathrm{~Hz}$, we took the average amplitude at 7.4, 7.5 and $7.6 \mathrm{~Hz}$ ). $S N R_{i}$ was obtained in Fourier power spectrum as the mean power of 3 bins centered around the harmonic frequency (e.g., mean power at $7.4,7.5$, and $7.6 \mathrm{~Hz}$ ) divided by the mean power of the surrounding 4 bins (e.g., mean power at 7.1, 7.2, 7.8 and $7.9 \mathrm{~Hz}$; two immediately adjacent bins were excluded). As the SSVEP responses in the present study were confined to $\mathrm{Oz}, \mathrm{O} 1$, and $\mathrm{O} 2$ electrodes (Fig. 5B), averaged responses at these 3 electrodes were used for further analysis.

The same analyses were carried out in Experiment 2 and Experiment 3 except that only 2 harmonics were considered instead of 5 . This was necessary because the decreased separation between the 8 frequencies in Experiment 2 meant that the 3rd or larger harmonics of some frequencies could overlap (e.g., the $3 \mathrm{rd}$ harmonic of $13.3 \mathrm{~Hz}$ and the 4th harmonic of $10 \mathrm{~Hz}$ are both at $40 \mathrm{~Hz}$ ). In Experiment 3, we only considered the first 2 harmonics as the 3rd harmonic $(60 \mathrm{~Hz}$ and $72 \mathrm{~Hz})$ of the flicker is too high in frequency and only adds extra noise and negligible SSVEP signals if included. We did an additional analysis in Experiment 3 to examine the contribution of saccades to the result (Fig. 3). In this analysis, we discarded EEG signals in a temporal window of $[-250250]$ ms locked to saccade onset by multiplying them with an inversed Tukey window (alpha $=0.33$ ). The temporal window of [ -250250$] \mathrm{ms}$ was chosen as it covered the period of peri-saccadic change in visual perception reported in almost all the literature (e.g., Ibbotson and Krekelberg, 2011; Rolfs et al., 2011; Li et al., 2016). As a result, EEG signals around saccades were set equal to zero and did not contribute to the SSVEP result. In the result, we reported effect size measurements, partial $\eta_{p}^{2}$ for ANOVA and Cohen's $d_{z}$ (Cohen, 1988) for within-subject $t$-test.

\section{Results}

\subsection{Gaze positions relative to target}

The position of gaze relative to the target is a possible source of confounds in this paradigm. Suppose the gaze led the target, the flickering stimulus in front of the target would have been viewed at a smaller eccentricity on the retina relative to the checkerboard in the 


\section{Exp 1}

Fixation

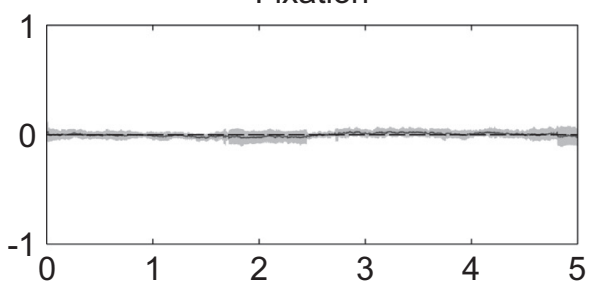

Exp 2

Fixation

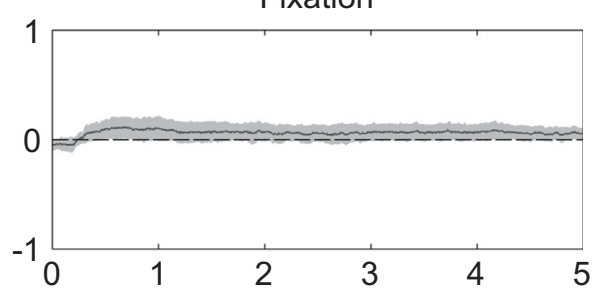

Fig. 4. Gaze position error (distance between gaze and target position) in each condition in Experiment 1 - two locations and Experiment 2 - multiple locations. Positive values indicate lead of gaze to target, whereas negative values indicate lags. Shaded area shows inter-participant SE.
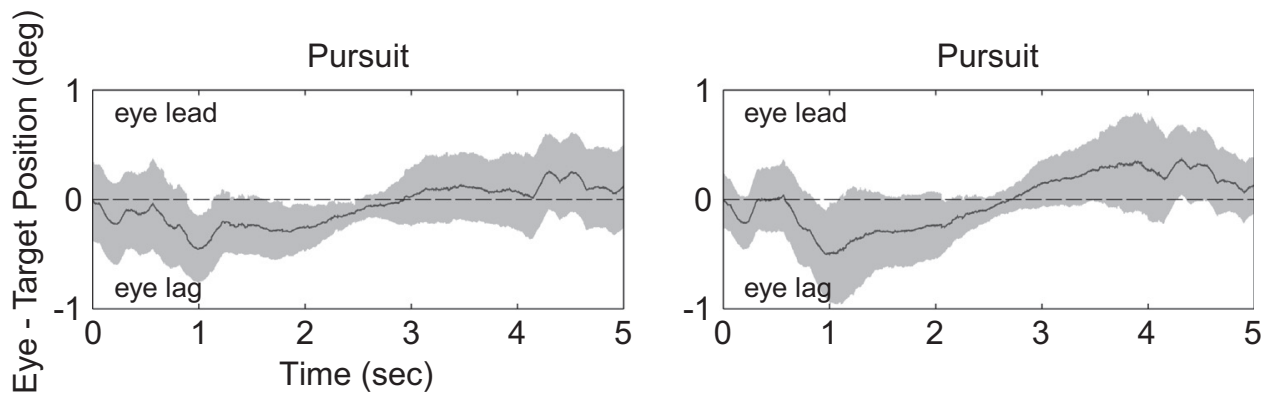

Attend while Fixating

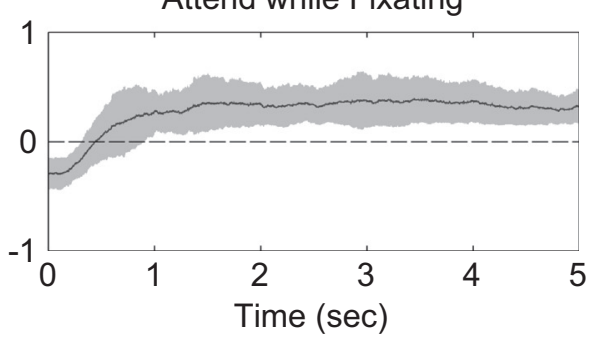

back, leading to higher SSVEP responses for the first compared to the latter.

In Experiment 1 - two locations, gaze was on average centered on target during fixation. During pursuit, the gaze on average was centered on the target, although it lagged the target within the first half of the epoch and slightly led in the second half. In Experiment 2 - multiple locations, during the fixation condition gaze was slightly in front of the simulated direction of pursuit. This was most likely due to a bottom-up capture by the cue that was to be used later in the attend-while-fixating condition. As in Experiment 1, during pursuit the gaze was on average centered on the target, lagging behind within the first half, and leading in the second half. In the attend-while-fixating condition, gaze was evidently shifted towards the attended side (Fig. 4).

\subsection{Enhanced SSVEP amplitudes in front of the pursuit target}

For Experiment 1 - two locations, the grand-average amplitude spectrum is shown in Fig. 5. The peaks at $6.7 \mathrm{~Hz}, 7.5 \mathrm{~Hz}$ and their harmonics are clearly visible. Notably, in pursuit trials, the amplitude at $6.7 \mathrm{~Hz}$ was higher when $6.7 \mathrm{~Hz}$ flicker was placed in the front of the target (green trace) than when it was placed in the back (dashed black). The same was true for $7.5 \mathrm{~Hz}$, the amplitude was higher when the 7.5 Hz flicker was in front of the target (dashed black) than when it was in the back (green trace). The topographic map of this enhancement was shown in Fig. 5B. The enhancement effect was exclusively located at occipital electrodes (O1, Oz and O2). For fixation trials (Fig. 5C, D), there was no sign of enhancement in SSVEP responses.

SSVEP amplitudes were labeled by the location of the corresponding stimulus relative to the pursuit direction (front vs. back) for pursuit trials. For fixation trials, where the whole stimulation pattern was replayed without pursuit, SSVEP amplitudes were correspondingly labeled as "front" and "back" as if pursuit had been actually happening. A
2 (condition: pursuit vs. fixation) $\times 2$ (location: front vs. back) repeated measure ANOVA revealed a main effect of location, $F(1,11)=$ 7.12, $P=0.022, \eta_{p}^{2}=0.39$, and an interaction between condition and location, $F(1,11)=6.12, P=0.026, \eta_{p}^{2}=0.38$. In the pursuit condition, SSVEP amplitude was higher in front (mean \pm SD, $0.228 \pm 0.075 \mu \mathrm{V})$ than in the back $(0.209 \pm 0.071), \mathrm{t}(11)=3.13, P=$ 0.0096 , Cohen's $d_{z}=0.90$ (Fig. 6A). In fixation condition, there was no significant difference (front: $0.231 \pm 0.079$; back: $0.230 \pm 0.071$ ), $t(11)=0.35, P=0.73$, Cohen's $d_{z}=0.10$ (Fig. 6B).

In Experiment 2 - multiple locations, we concurrently tagged 8 locations ( 4 on the horizontal axis, 4 on the vertical axis, Fig. 1B). Fig. 7 shows the grand-average of the amplitude spectrum of the EEG responses. The clear peaks at 8 tagged frequencies (marked by green arrows) indicate that our experiment design did manage to tag the SSVEP responses to individual flickers. EEG amplitude in the alpha range $(8-12 \mathrm{~Hz})$ decreased from the fixation condition, to the "attend-whilefixating" condition, to the pursuit condition. Notice that this was also the case in Experiment 1, where alpha power in the pursuit condition was decreased compared to the fixation condition (Fig. 5, decreased alpha power between A and C). This probably was because alpha power tends to decrease with increasing task demands (Klimesch, 1999). However, note that the difference in alpha power between the 3 conditions did not make any impact on our main results, as the frequencies were all counterbalanced and the comparisons of interests were asymmetry effects within each condition.

We quantified SSVEP amplitudes in each condition and at both $1.5^{\circ}$ \% $3.5^{\circ}$ eccentricities. For the horizontal 4 locations, two sides were labeled as front/back based on the pursuit direction (or simulated pursuit direction in fixation condition), or as attended/unattended based on the attention (Fig. 8). A 3 (condition: fixation vs. pursuit vs. attend-whilefixating) $\times 2$ (location: front/attended vs. back/unattended) $\times 2$ (eccentricity: $1.5^{\circ}$ vs. $3.5^{\circ}$ ) repeated measures ANOVA over the SSVEP 
A

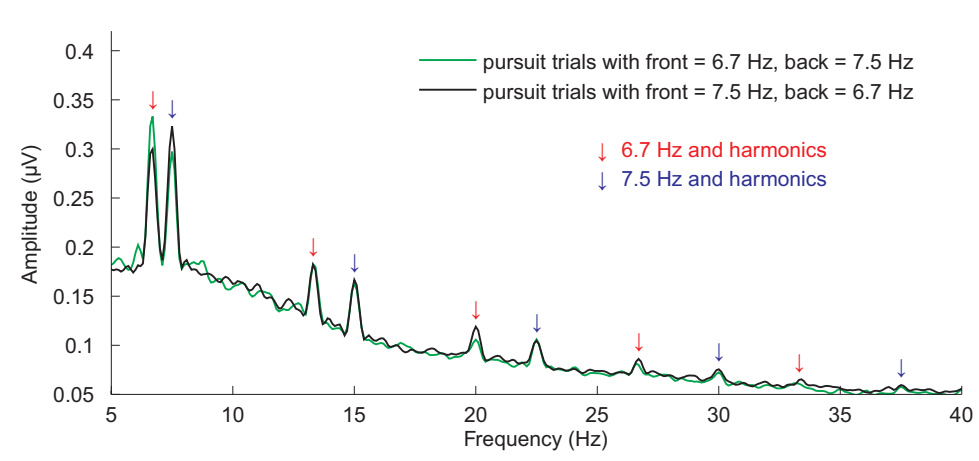

C

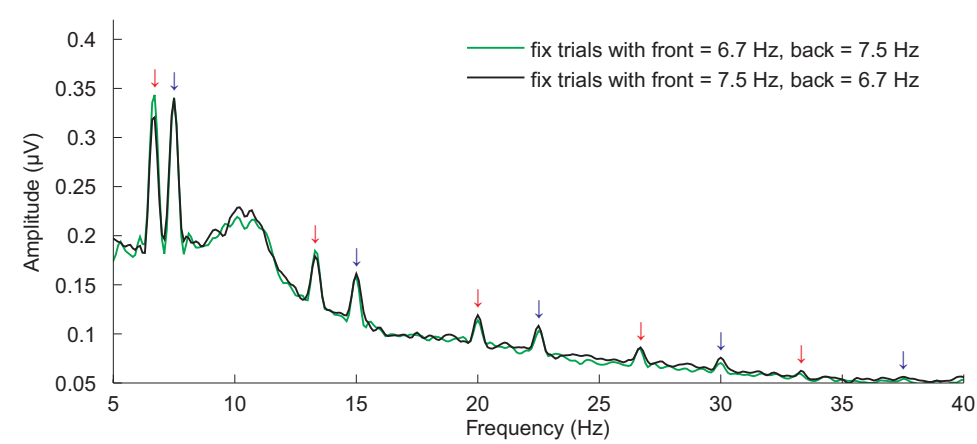

B

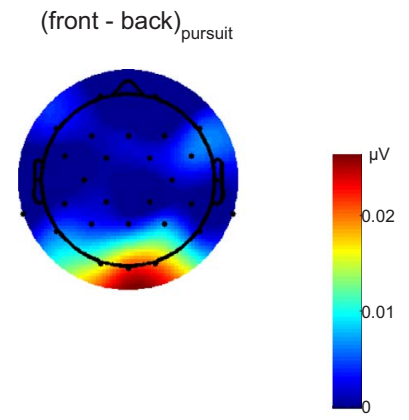

D (front - back) $)_{\text {fixation }}$

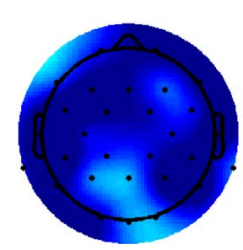

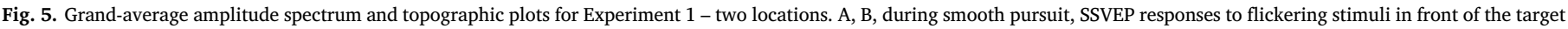
were enhanced compared to stimuli behind. C, D, during fixation, SSVEP responses were equivalent.

amplitudes revealed a main effect of condition, $F(2,22)=4.85, P=$ $0.018, \eta_{p}^{2}=0.31$, a main effect of location, $F(1,11)=14.78, P=$ $0.003, \eta_{p}^{2}=0.57$, an interaction between condition and location, $F(2,22)=5.50, P=0.02, \eta_{p}^{2}=0.33$, and an interaction between location and eccentricity, $F(1,11)=17.90, P=0.001, \eta_{p}^{2}=0.62$. Subsequently, we examined the effect separately in each condition. In fixation condition, we did not observe significant main effect of location $(P=0.16)$, main effect of eccentricity $(P=0.71)$ nor their interaction $(P=0.30)$. In pursuit condition, we found a significant interaction between location and eccentricity, $F(1,11)=16.35, P=0.002, \eta_{p}^{2}=$ 0.60 . At $1.5^{\circ}$ eccentricity, the front location had a higher SSVEP response (mean $\pm \mathrm{SD}, 0.205 \pm 0.049 \mu \mathrm{V}$ ) than the back location $(0.188 \pm 0.041), t(11)=3.10, P=0.01$, Cohen's $d_{z}=0.89$. At $3.5^{\circ}$ eccentricity, there was no difference (front: $0.183 \pm 0.064$; back: $0.186 \pm 0.062), t(11)=0.80, P=0.44$, Cohen's $d_{z}=-0.23$. In the

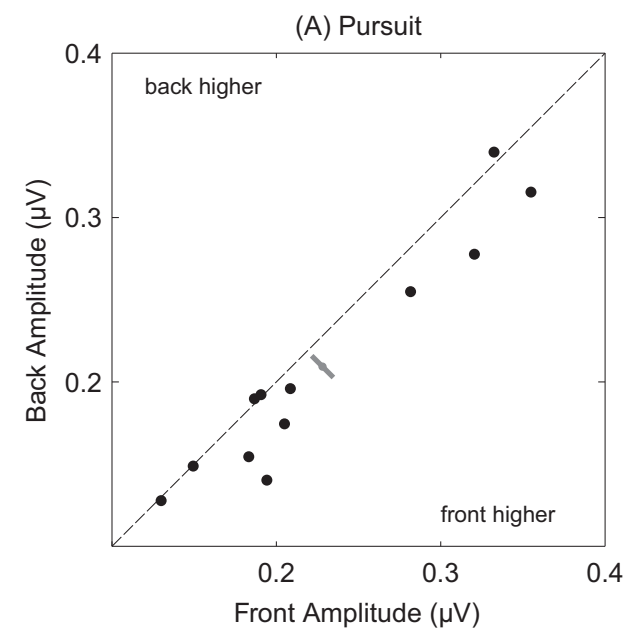

attend-while-fixating condition, we observed a main effect of location, $F(1,11)=15.19, P=0.002, \eta_{p}^{2}=0.58$; and an interaction between location and eccentricity, $F(1,11)=9.09, P=0.012, \eta_{p}^{2}=0.45$. At $1.5^{\circ}$ eccentricity, the attended side elicited a higher SSVEP amplitude $(0.224 \pm 0.050)$ than the unattended side $(0.192 \pm 0.037), t(11)=$ 3.80, $P=0.003$, Cohen's $d_{z}=1.10$. At $3.5^{\circ}$ eccentricity, the attended side also produced a higher response $(0.210 \pm 0.073)$ than the unattended side $(0.199 \pm 0.069), t(11)=2.52, P=0.028$, Cohen's $d_{z}=$ 0.73 .

We observed an SSVEP enhancement at $3.5^{\circ}$ eccentricity in the attend-while-fixating condition, but not in the pursuit condition. One may argue that, instead of due to attention, this might be due to the fact that the gaze tended to shift in the direction of the cue in this condition (Fig. 4). We thus correlated the gaze shift with the SSVEP enhancement at $3.5^{\circ}$ eccentricity across observers, and found a moderate but nonsignificant correlation, $\mathrm{r}=0.32, P=0.30$. The moderate correlation

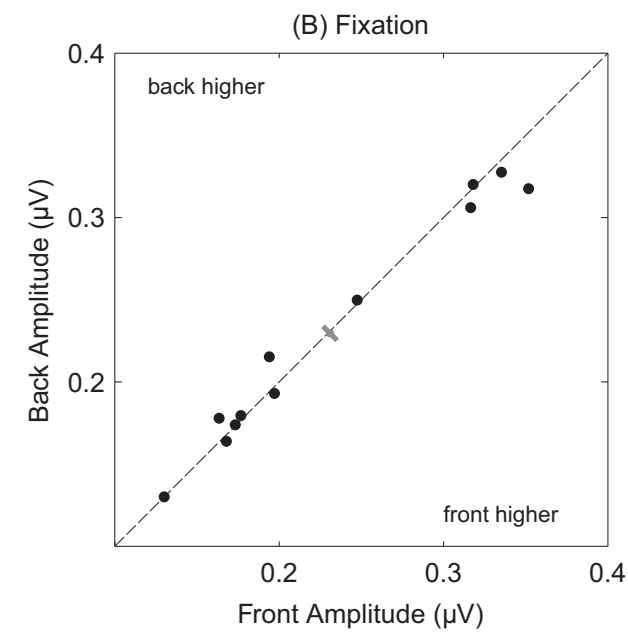

Fig. 6. SSVEP amplitude of the flicker frequencies of the stimulus at the back of the target compared against that from the stimulus in front for individual observers, for Experiment 1 - two locations. A, smooth pursuit trials. B, fixation trials. The front and back flickering checkerboards in fixation trials were defined considering the stimulus as a replay of a pursuit trial. Results shows a higher amplitude for the stimulus in front during pursuit, but not during fixation. Filled circles indicate individual observers. Gray bars show the $95 \%$ confidence intervals of the mean along the negative-slope diagonal line. 


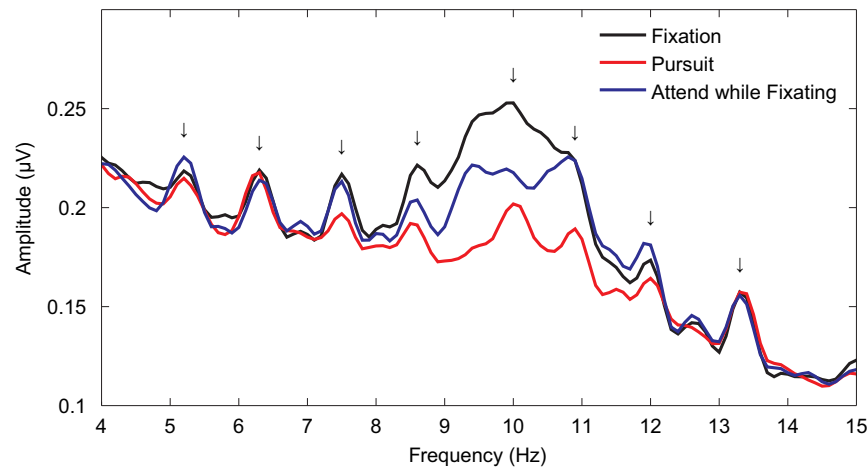

Fig. 7. Grand-average amplitude spectrum for 3 conditions (fixation, pursuit, and attendwhile-fixating), for Experiment 2 - multiple locations. The clear peaks at tagged frequencies (marked by green arrows: 5.2, 6.3, 7.5, 8.6, 10.0, 10.9, 12.0, 13.3 Hz) shows that our design did manage to isolate SSVEP responses to 8 flickers.

may imply a small contribution of gaze shift to the SSVEP enhancement. It is, however, well established that spatial attention enhances SSVEP responses to periphery stimuli (e.g., at $5.7^{\circ}$ in Morgan et al., 1996; at $7^{\circ}$ in Störmer et al., 2014). Therefore, we are confident that the SSVEP enhancement at $3.5^{\circ}$ was mainly the result of spatial attention rather than overt gaze shifts in the attend-while-fixating condition. In contrast, the fact that SSVEP was not enhanced at $3.5^{\circ}$ for pursuit condition means that the attentional bias at $3.5^{\circ}$ eccentricity was too weak to induce any SSVEP enhancement.

The SSVEP amplitudes along the vertical axis are shown in Fig. 9. A 3 (condition) $\times 2$ (visual hemifield: top vs. bottom) $\times 2$ (eccentricity: 1.5 vs. $3.5^{\circ}$ ) repeated measure ANOVA revealed a main effect of condition, $F(2,22)=6.35, P=0.011, \eta_{p}^{2}=0.37$. SSVEP amplitudes were overall highest in fixation condition, and lowest in pursuit condition (the average in fixation: $0.209 \mu \mathrm{V}$; fixation-while-attending: 0.203 ; pursuit: 0.193; with the difference between fixation and pursuit being significant, $\mathrm{P}=0.04$ ). This was most likely due to the difference in alpha power between 3 conditions (Fig. 7). There was a main effect of visual hemifield, $F(1,11)=22.92, P=0.001, \eta_{p}^{2}=0.68$, with SSVEP amplitudes from stimuli in the lower hemifield higher than those from the upper hemifield. There was a significant main effect of eccentricity, $F(1,11)=18.97, P=0.001, \eta_{p}^{2}=0.63$; and a significant interaction between visual hemifield and eccentricity, $F(1,11)=11.07, P=$ $0.007, \eta_{p}^{2}=0.50$. These effects suggest a top-bottom asymmetry of SSVEP responses that probably reflects the fact that EEG Oz/O1/O2 electrodes pick up more signals from the lower hemifield of the fovea
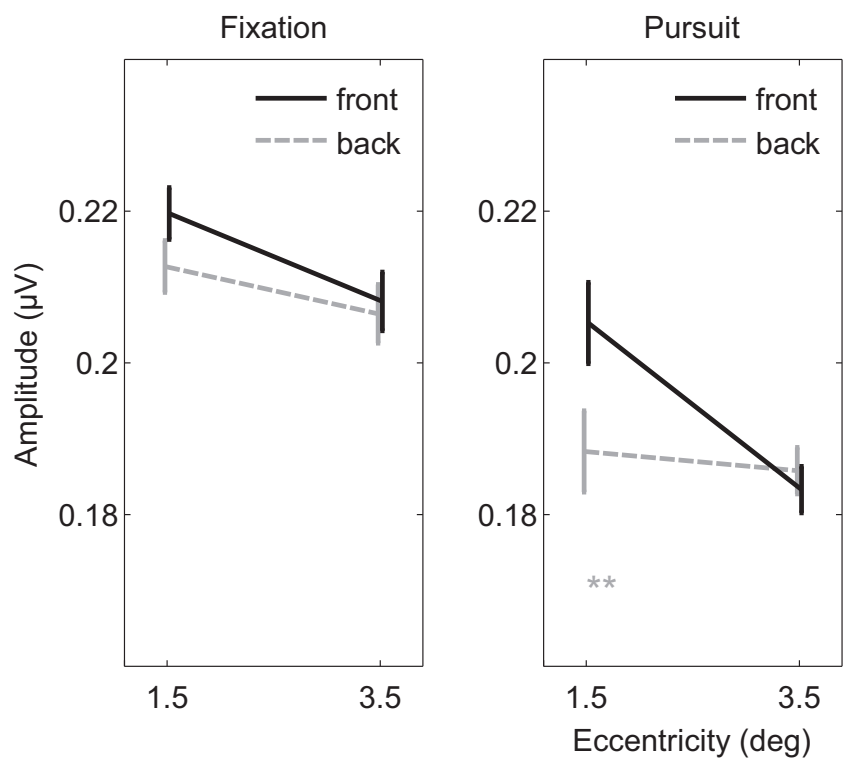

due to the retinotopic mapping in primary visual cortex, which is out of the scope of the current paper. Of primary interest, we failed to find any significant interaction between condition and other two variables (condition with visual hemifield, $P=0.18$; condition with eccentricity, $P=0.56$; or condition with both, $P=0.22$ ). It appears that pursuing a target and orienting visuospatial attention along the horizontal axis hardly changes the allocation of attention along the vertical axis.

\subsection{No contribution of pursuit gaze errors to the SSVEP enhancement}

During smooth pursuit, the gaze on average lagged behind the target in the 1st half of pursuit and led in front of the target in the 2nd half in both Experiment 1 - two locations and Experiment 2 multiple locations (Fig. 4). Did this gaze lead in the 2nd half cause the SSVEP enhancement we observed? We separately analyzed SSVEP responses in the 1st and 2nd half of each epoch during smooth pursuit. In Experiment 1, a 2 (signal location: 1st half vs. 2 nd half) $\times 2$ (flicker location: front vs. back) ANOVA over the SSVEP amplitude revealed a marginal main effect of flicker location, $F(1,11)=3.92, P=0.073$, without interaction between 2 factors, $F(1,11)=1.12, P=0.31$, suggesting that the 1st and 2nd half did not differ in the SSVEP enhancement. In fact, should there be any difference, a significant SSVEP enhancement was observed for the 1st half epoch, $t(11)=2.50, P=$ 0.029 , Cohen's $d_{z}=0.72$, but was not for the 2nd half, $t(11)=1.06$, $P=0.31$, Cohen's $d_{z}=0.31$. Therefore, the small gaze lead in the 2nd half of each epoch did not contribute significantly to the SSVEP enhancement we observed. In Experiment 2 - multiple locations, a 2 (signal location: 1 st half vs. 2nd half) $\times 2$ (flicker location: front vs. back) ANOVA over the SSVEP amplitude at $1.5^{\circ}$ eccentricity revealed a marginally significant main effect of flicker location, $F(1,11)=3.82$, $P=0.076$, without interaction between two factors, $F(1,11)=0.052$, $P=0.82$. If tested separately, SSVEP enhancement was approaching significance in both the 1st half signal, $t(11)=2.01, P=0.069$, Cohen's $d_{z}=0.58$, and the 2nd half signal, $t(11)=1.56, P=0.15$, Cohen's $d_{z}=0.45$. Taken together, results from Experiment 1 and 2 concur to indicate that gaze errors during smooth pursuit had little contribution to the observed SSVEP enhancement.

\subsection{No effect of catch-up saccades during pursuit on SSVEP amplitude}

The known attentional enhancement towards saccade target may contribute to the SSVEP enhancement during smooth pursuit, as catchup saccades occur frequently. During the middle $3 \mathrm{~s}$ in each epoch, the

\section{Attend while Fixating}

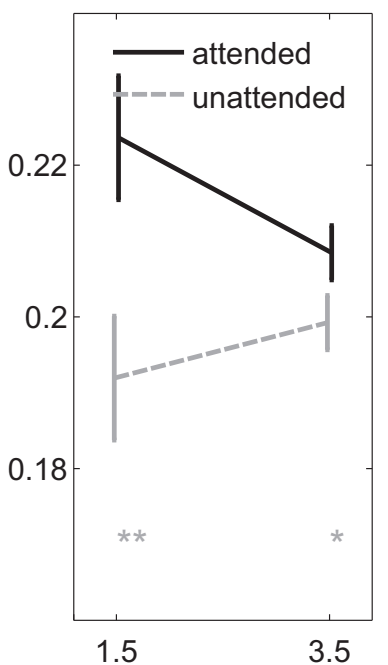

Fig. 8. Amplitudes of SSVEP responses in 3 conditions at both $1.5^{\circ}$ and $3.5^{\circ}$ eccentricities for Experiment 2 - multiple locations. In Fixation condition, the front/back was labeled by treating the trial as a pursuit trial. Enhanced responses to front flickers was observed at only $1.5^{\circ}$ eccentricity for Pursuit condition, while the enhancement was present at both $1.5^{\circ}$ and $3.5^{\circ}$ eccentricity for "attentionwhile-fixating" condition. Error bars represent within-participant $95 \%$ confidence interval (Cousineau, 2005). ${ }^{*} P<0.05,{ }^{*} P<0.01$. 

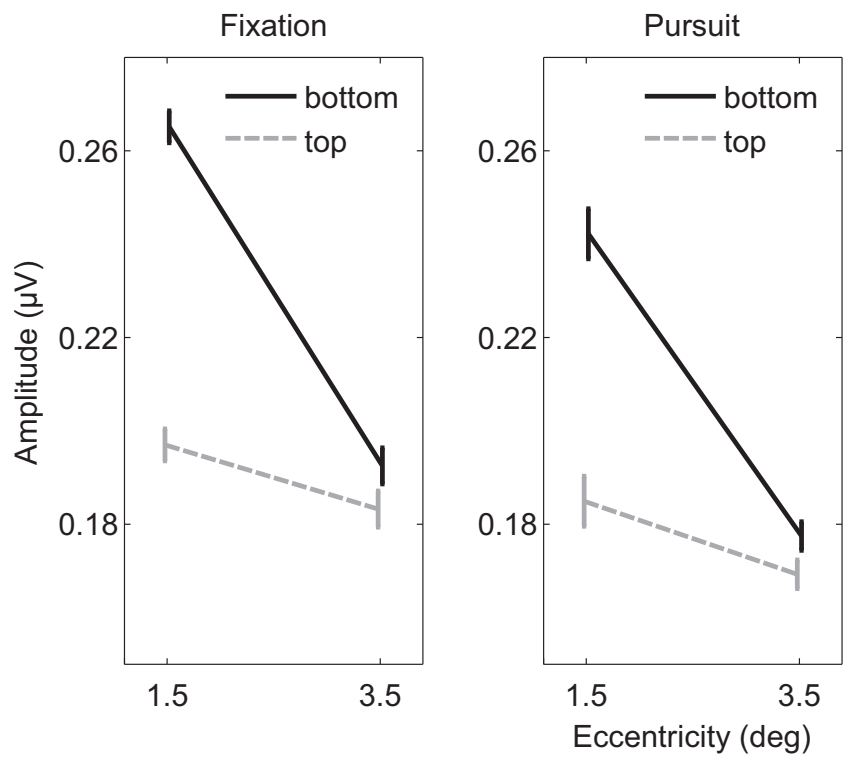

average number of saccades was 3.81 in Experiment 1 - two locations and 4.35 in Experiment 2 - eight locations. The large amount of saccades and the low flickering frequencies made it difficult to investigate the contribution of saccades to the SSVEP enhancement in Experiment 1 and 2. In Experiment 3 - effect of saccades, we modified the design so that participants made fewer saccades ( 2.75 saccades during the middle $3 \mathrm{~s}$ ). The flickering frequencies were $20 \mathrm{~Hz}$ and $24 \mathrm{~Hz}$, which allowed us to perform a fast Fourier analysis on shorter duration of EEG signals after excluding the part where saccades occurred.

We first tried to replicate the SSVEP enhancement effect in Experiment 3 - effect of saccades, using the same analysis as that in Experiment 1 and 2. SSVEP amplitude was higher in front of the pursuit target than in the back, $t(15)=2.18, P=0.046$. Cohen's $d_{z}=0.54$. In order to access the contribution of saccades to SSVEP, we took away the EEG signals around each saccade $(-250 \mathrm{~ms}$ before to $250 \mathrm{~ms}$ after saccade onset) by windowing. We still observed the SSVEP enhancement effect (Fig. 10), $t(15)=3.07, P=0.0077$, Cohen's $d_{z}=0.77$. Therefore, the result held even though we did not take into account the EEG signals around saccades.

\section{Discussion}

In the current study, we measured frequency-tagged SSVEP responses to flickering stimuli located at various positions relative to the

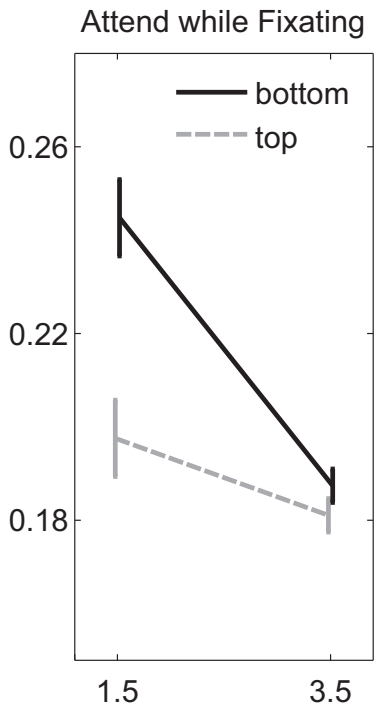

Fig. 9. SSVEP response amplitude from stimuli along the vertical axis for 3 conditions and at $1.5^{\circ} / 3.5^{\circ}$ eccentricity for Experiment 2 - multiple locations. All three conditions showed the same pattern. Error bars represent within-participant $95 \%$ confidence interval (Cousineau, 2005).

target during slow smooth pursuit eye movements. We found an enhanced SSVEP response for the flickering stimulus located closely in front of the pursuit target, compared to the flickering stimulus located in the back. Our analyses ruled out the possibility that the SSVEP enhancement was a byproduct of the catch-up saccades occurring during pursuit and a possible confound in terms of gaze leading the target during pursuit. The enhancement reveals a default attentional bias towards the direction of smooth pursuit eye movements.

\subsection{Attention is allocated closely ahead of the pursuit target}

Our result is consistent with previous studies showing that saccadic or manual reaction times are faster to a target in the pursuit direction than for one in the opposite direction (Tanaka et al., 1998; van Donkelaar, 1999; van Donkelaar and Drew, 2002; Kanai et al., 2003; Blohm et al., 2005; Khan et al., 2010; Seya and Mori, 2012), whereas it is in conflict with findings that perceptual discrimination accuracy is symmetric along the pursuit direction (Lovejoy et al., 2009; Watamaniuk and Heinen, 2015). In these studies (Lovejoy et al., 2009; Watamaniuk and Heinen, 2015), the potential locations of the targets for the perceptual discrimination task were visible throughout the trial. Participants probably distributed attention equally to these potential locations, overriding any default bias (as discussed in Watamaniuk and Heinen, 2015).

A

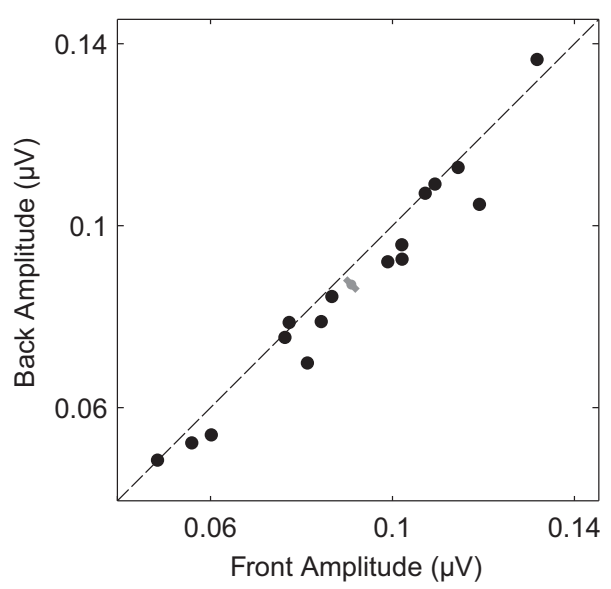

B

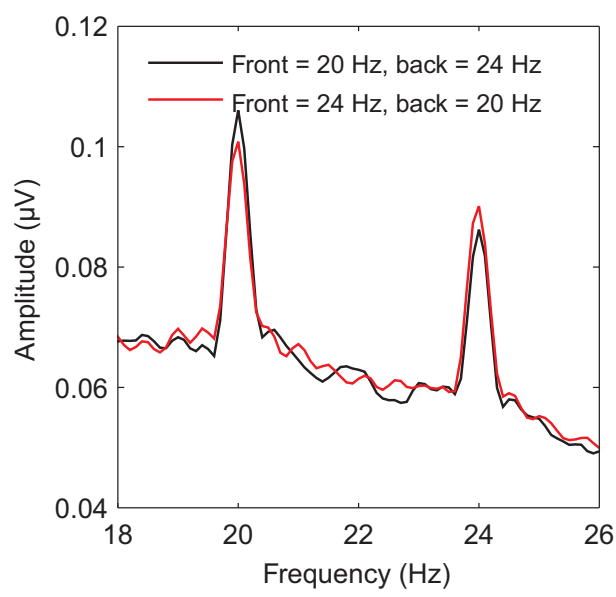

Fig. 10. SSVEP results of the EEG signals after removing saccade-related signals in Experiment 3 effects of saccades. A, SSVEP amplitude of the flicker at the back of the target compared against that of the flicker in front for individual observers, showing an enhanced SSVEP responses to the flickering stimulus in front. Gray bar shows the $95 \%$ confidence interval of the mean along the negative-slope diagonal line. $\mathrm{B}$, amplitude spectrum for trials with $20 \mathrm{~Hz}$ flicker in front (dark line) and trials with $24 \mathrm{~Hz}$ flicker in front (red line). Clearly, amplitude at the frequency in front was enhanced. 
In addition, our result differs from some previous studies on the spatial extension of the attentional enhancement during smooth pursuit. We observed SSVEP enhancement only at $1.5^{\circ}$ eccentricity but not at $3.5^{\circ}$, suggesting that the attentional bias is just closely ahead. In contrast, modulation of saccadic/manual reaction times has been observed up to more than $10^{\circ}$ ahead (Tanaka et al., 1998; Khan et al., 2010; Seya and Mori, 2012), leading to the view that attentional bias spreads to the entire visual field ahead of pursuit (Khan et al., 2010). Nevertheless, modulation of saccadic reaction times could also be a result of interactions between saccadic system and smooth pursuit system (e.g., proposed in Tanaka et al., 1998), given the overlap in neural controls for saccades and pursuit (Basso et al., 2000; Krauzlis, 2003, 2004; Orban de Xivry and Lefèvre, 2007). The same is true for manual response, as previous studies revealed close synergies between smooth pursuit and hand movements (Maioli et al., 2007; Hiraoka et al., 2014; Niehorster et al., 2015; Chen et al., 2016a, 2016b). Therefore, due to interactions between different motor commands, tasks requiring reaction to probe stimuli are not ideal for investigating attention during smooth pursuit eye movements. Instead, an index related to early visual processing might be preferred. Interestingly, Harrison et al. (2014) investigated the effect of smooth pursuit on visual crowding, a phenomenon likely due to processing constraints in primary visual cortex (Pelli, 2008). They observed less crowding closely ahead in the direction of pursuit but not further ahead. As attention reduces crowding (Yeshurun and Rashal, 2010), their results may imply enhanced attention closely in front of pursuit. Taken together, smooth pursuit eye movements likely induce an attentional bias closely ahead in the direction of pursuit. A related question is whether the location of attentional enhancement may vary with pursuit speed. Khan et al. (2010)'s behavioral study suggests that it does. With higher pursuit speed, the enhancement seemed to occur at a location further in front of the pursuit target. We would predict that SSVEP enhancement may also occur at a further location with increasing pursuit velocity.

The enhancement in SSVEP induced by pursuit is slightly smaller than for the case when attention is explicitly directed to the target (Fig. 8). This is consistent with previous findings that attentional effects are usually not very strong during smooth pursuit (e.g., Kerzel et al., 2008). It should be noted though that the effect was highly reliable and robust across three versions of the experiment, and that the actual effect size was large (Cohen's $d_{z}=0.90,0.89,0.77$, in the three experiments, respectively).

Another striking feature of our data is a strong top-bottom asymmetry in the SSVEP under all conditions. There are two potential reasons for this. First, this could be a consequence of the retinotopy in V1, where the lower hemifield projects to the upper part of V1. The occipital electrodes $(\mathrm{Oz} / \mathrm{O} 1 / \mathrm{O} 2)$ are therefore closer to the lower hemifield projection and pick up a stronger signal. This leads to a larger response for lower hemifield stimuli (see discussion in Hagler, 2014). Second, attentional factors may also play a role here, as previous studies have reported attentional facilitation for the lower hemifield (He et al., 1996; Carrasco et al., 2001). Of course, the most important aspect of our data, the differential distribution of attention along the path of pursuit, is independent of any bias in the baseline distribution of attention.

The present study used neurophysiological markers to infer attention during smooth pursuit, whereas previous related studies used behavioral measurements (reaction times or perceptual discrimination accuracy). It will be interesting to see in further studies to what degree SSVEP and the various behavioral measures of attention deployment during smooth pursuit are measuring the same underlying processes.

\subsection{Smooth pursuit and the premotor theory of attention}

According to the premotor theory of attention, shifts of attention are consequences of planning movements (reach or eye movements) towards intended locations (Rizzolatti et al., 1987). Although the theory is controversial (Smith and Schenk, 2012; Belopolsky and Theeuwes,
2012; MacLean et al., 2015), saccades and attention do share neural mechanisms in some cortical areas (Corbetta et al., 1998; Moore and Armstrong, 2003) and in the superior colliculus (SC, McPeek and Keller, 2004; Carello and Krauzlis, 2004; Müller et al., 2005). The generation of saccades involves a drop in the activity of fixation neurons and a buildup of activity in saccadic neurons in the frontal eye fields and SC (Dorris and Munoz, 1995; Dorris et al., 1997; Everling and Munoz, 2000). Activity of saccadic neurons at a given position of the topographical map in the SC can lead to a saccade characterized by a certain direction and amplitude. It has been found that the SC also plays a role in the control of smooth pursuit (Basso et al., 2000; Krauzlis and Dill, 2002; Krauzlis, 2003). Krauzlis (2003) suggested that the buildup activity of SC neurons gates pursuit initiation and triggers saccades as well. Overall, the control of saccadic eye movements, smooth pursuit and attention seems to be closely interacting (Krauzlis et al., 2013). Smooth pursuit eye movements may induce the attentional bias we observed in the present study as pursuit engages imbalanced activities in the direction of pursuit in the SC map. Alternatively, it could also be hypothesized that a shift of attention is necessary to drive the buildup of activity in SC to sustain smooth pursuit. Hafed, Goffart and Krauzlis (2008) have shown that SC activities during smooth pursuit is related to the coding of the intended spatial goal of the eye movement. This seems to conflict with our finding that the attentional enhancement in front of the target is present even when the eye is leading the target. The lead of the eye in the present study, however, was less than $0.5 \mathrm{deg}$. It is possible that a large enough lead of the eye that can trigger a backward corrective saccade could also lead to attention being allocated behind the pursuit target.

Studies on predictive remapping before saccades suggest that neurons pre-activate in anticipation of the consequence of saccades (Duhamel et al., 1992), and that attention remaps to the anticipated post-saccadic location as well (Rolfs et al., 2011). For smooth pursuit, a recent study (Dowiasch et al., 2016) recorded neuron activities in the ventral intraparietal area (VIP), and observed anticipatory activations in the direction of pursuit. Whether VIP forms the neural basis for the attentional bias in smooth pursuit requires further investigations.

\subsection{Why attention is closely in front of pursuit target}

While it is possible that the attentional bias induced by pursuit is a side effect due to the shared neural controls, it might actually serve a functional role. One advantage of pursuit is, of course, to enable inspection of the moving object with high acuity (Schütz et al., 2009). However, there are other important aspects (see Gegenfurtner, 2016). For example, pursing a moving object leads to better prediction of the future motion trajectory and improved manual interception (Brenner and Smeets, 2009, 2011; Spering et al., 2011; Fooken et al., 2016). It is thus beneficial to allocate attention in front of pursuit along the future path of motion.

Furthermore, it is known that feed-forward processes are too slow to drive smooth pursuit because of neural delays, and top-down predictive mechanisms are necessary to sustain smooth pursuit (e.g., Krauzlis and Lisberger, 1994). Selectively attending ahead of the pursuit target may pull the gaze towards the front direction (Hafed and GoffartandKrauzlis, 2008), countering the lag in the visuo-oculomotor loop and allowing for more accurate pursuit. Attention must be allocated in an anticipatory manner so as to drive predictive smooth pursuit and predictive saccades (Hayhoe et al., 2012; Fooken et al., 2016; for reviews, see Kowler, 2011; Kowler et al., 2014).

\subsection{Studying attention and perception during eye movements with SSVEP}

SSVEP has been used to study low-level visual processes (e.g., Norcia and Tyler, 1985), attention (Andersen et al., 2011), and recently higher-level perception such as face perception (Rossion and Boremanse, 2011). We argue that it also constitutes an additional 
powerful technique next to those that have been used to study perception during eye movements in the past (Schütz et al., 2011). First, SSVEP allows tagging multiple locations concurrently, avoiding any potential fluctuations of attention across trials/secessions. Second, the procedure is implicit without any secondary probing task on top of eye movement execution, thus avoiding potential biases due to the probing task. Third, in contrast to the event-related potential (ERP), the SSVEP technique is rather immune to the electrooculogram (EOG) artifacts from eye movements, as signals not phase-locked to the flickering stimuli are discarded by the spectrum analysis. There are, however, limitations of the SSVEP technique. For example, the temporal resolution of the SSVEP is worse compared to ERPs. In addition, given the noisy nature of EEG signals, the usefulness of the SSVEP technique is constrained by its signal-to-noise ratio.

\subsection{Conclusion}

Our result that SSVEPs elicited by stimuli in front of the pursuit target are enhanced, relative to stimuli behind it, clearly shows that attention is allocated slightly ahead of the fixation point during pursuit. Our results provide new evidence about the allocation of attention during smooth pursuit eye movements. The previous findings had been inconsistent. We present the first electrophysiological evidence and it supports the notion that attention is on average allocated ahead of the pursuit target. SSVEPs seem to be a powerful technique to monitor attention allocation during continuous eye movements without the need for an overt task.

\section{Acknowledgements}

We thank Pierre-Pascal Forster for help with EEG data collection, and Maria Ayala for help with proofreading the manuscript. All the data and experimental scripts are available at http://doi.org/10.5281/ zenodo.817545. J. Chen was supported by the Deutsche Forschungsgemeinschaft (DFG) International Research Training Group IRTG 1901, "The Brain in Action". M. Valsecchi was supported by the DFG SFB TRR 135, "Cardinal Mechanisms of Perception" and by the European Union Marie Curie Initial Training Network "PRISM" (FP7PEOPLE-2012-ITN; grant agreement 316746).

\section{References}

Andersen, Sr.K., Müller, M.M., Hillyard, S.A., 2011. Tracking the allocation of attention in visual scenes with steady-state evoked potentials. Cogn. Neurosci. Atten. 2, 197-216.

Awh, E., Armstrong, K.M., Moore, T., 2006. Visual and oculomotor selection: links, causes and implications for spatial attention. Trends Cogn. Sci. 10, 124-130.

Bach, M., Meigen, T., 1999. Do's and don'ts in Fourier analysis of steady-state potentials. Documenta Ophthalmologica 99, 69-82.

Basso, M.A., Krauzlis, R.J., Wurtz, R.H., 2000. Activation and inactivation of rostral superior colliculus neurons during smooth-pursuit eye movements in monkeys. J. Neurophysiol. 84, 892-908.

Belopolsky, A.V., Theeuwes, J., 2012. Updating the premotor theory: the allocation of attention is not always accompanied by saccade preparation. J. Exp. Psychol.: Human. Percept. Perform. 38, 902.

Blohm, G., Missal, M., Lefevre, P., 2005. Processing of retinal and extraretinal signals for memory-guided saccades during smooth pursuit. J. Neurophysiol. 93, 1510-1522.

Brainard, D.H., 1997. The psychophysics toolbox. Spatial Vision 10, 433-436.

Brenner, E., Smeets, J.B., 2009. Sources of variability in interceptive movements. Exp. brain Res. 195, 117-133.

Brenner, E., Smeets, J.B., 2011. Continuous visual control of interception. Human. Mov. Sci. 30, 475-494.

Carello, C.D., Krauzlis, R.J., 2004. Manipulating intent: evidence for a causal role of the superior colliculus in target selection. Neuron 43, 575-583.

Carrasco, M., Talgar, C.P., Cameron, E.L., 2001. Characterizing visual performance fields: effects of transient covert attention, spatial frequency, eccentricity, task and set size. Spat. Vision. 15, 61-75.

Chen, C.-Y., Ignashchenkova, A., Thier, P., Hafed, Z.M., 2015. Neuronal response gain enhancement prior to microsaccades. Curr. Biol. 25, 2065-2074.

Chen, J., Valsecchi, M., Gegenfurtner, K.R., 2016a. LRP predicts smooth pursuit eye movement onset during the ocular tracking of self-generated movements. J. Neurophysiol. 116, 18-29.

Chen, J., Valsecchi, M., Gegenfurtner, K.R., 2016b. Role of motor execution in the ocular tracking of self-generated movements. J. Neurophysiol. 116, 2586-2593.
Cohen, J., 1988. Statistical Power Analysis for the Behavioral Sciences. Routledge.

Corbetta, M., Akbudak, E., Conturo, T.E., Snyder, A.Z., Ollinger, J.M., Drury, H.A., Linenweber, M.R., Petersen, S.E., Raichle, M.E., Van Essen, D.C., 1998. A common network of functional areas for attention and eye movements. Neuron 21, 761-773.

Cousineau, D., 2005. Confidence intervals in within-subject designs: a simpler solution to Loftus and Masson's method. Tutor. Quant. Methods Psychol. 1, 42-45.

Delorme, A., Makeig, S., 2004. EEGLAB: an open source toolbox for analysis of single-trial EEG dynamics including independent component analysis. J. Neurosci. Methods 134 9-21.

Deubel, H., Schneider, W.X., 1996. Saccade target selection and object recognition: evidence for a common attentional mechanism. Vision. Res. 36, 1827-1837.

Dorr, M., Martinetz, T., Gegenfurtner, K.R., Barth, E., 2010. Variability of eye movements when viewing dynamic natural scenes. J. Vision. 10 (28-28).

Dorris, M.C., Munoz, D.P., 1995. A neural correlate for the gap effect on saccadic reaction times in monkey. J. Neurophysiol. 73, 2558-2562.

Dorris, M.C., Pare, M., Munoz, D.P., 1997. Neuronal activity in monkey superior colliculus related to the initiation of saccadic eye movements. J. Neurosci. 17, 8566-8579.

Dowiasch, S., Blohm, G., Bremmer, F., 2016. Neural correlate of spatial (mis-) localization during smooth eye movements. Eur. J. Neurosci. 44, 1846-1855.

Duhamel, J.-R., Colby, C.L., Goldberg, M.E., 1992. The updating of the representation of visual space in parietal cortex by intended eye movements. Science 255, 90.

Engbert, R., Kliegl, R., 2003. Microsaccades uncover the orientation of covert attention. Vision. Res. 43, 1035-1045.

Everling, S., Munoz, D.P., 2000. Neuronal correlates for preparatory set associated with pro-saccades and anti-saccades in the primate frontal eye field. J. Neurosci. 20, $387-400$.

Fooken, J., Yeo, S.-H., Pai, D.K., Spering, M., 2016. Eye movement accuracy determines natural interception strategies. J. Vision. 16 (1-1).

Gegenfurtner, K.R., 2016. The interaction between vision and eye movements. Perception $45,1333-1357$.

Gray, M.J., Frey, H.-P., Wilson, T.J., Foxe, J.J., 2015. Oscillatory recruitment of bilateral visual cortex during spatial attention to competing rhythmic inputs. J. Neurosci. 35, 5489-5503.

Hafed, Z.M., Goffart, L., Krauzlis, R.J., 2008. Superior colliculus inactivation causes stable offsets in eye position during tracking. J. Neurosci. 28, 8124-8137.

Hafed, Z.M., Lovejoy, L.P., Krauzlis, R.J., 2011. Modulation of microsaccades in monkey during a covert visual attention task. J. Neurosci. 31, 15219-15230.

Hagler, D.J., 2014. Visual field asymmetries in visual evoked responses. J. Vision. 14 (13-13).

Harrison, W., Remington, R., Mattingley, J., 2014. Visual crowding is anisotropic along the horizontal meridian during smooth pursuit. J. Vision. 14 (21-21).

Hayhoe, M.M., McKinney, T., Chajka, K., Pelz, J.B., 2012. Predictive eye movements in natural vision. Exp. brain Res. 217, 125-136.

He, S., Cavanagh, P., Intriligator, J., 1996. Attentional resolution and the locus of visual awareness. Nature 383, 334.

Hiraoka, K., Ae, M., Ogura, N., Komuratani, S., Sano, C., Shiomi, K., Morita, Y., Yokoyama, H., 2014. Smooth pursuit eye movement preferentially facilitates motorevoked potential elicited by anterior-posterior current in the brain. Neuroreport 25, 279-283.

Hoffman, J.E., Subramaniam, B., 1995. The role of visual attention in saccadic eye movements. Atten. Percept. Psychophys. 57, 787-795.

Hutton, S., Tegally, D., 2005. The effects of dividing attention on smooth pursuit eye tracking. Exp. Brain Res. 163, 306-313.

Ibbotson, M., Krekelberg, B., 2011. Visual perception and saccadic eye movements. Curr. Opin. Neurobiol. 21, 553-558.

Itti, L., 2005. Quantifying the contribution of low-level saliency to human eye movements in dynamic scenes. Vis. Cogn. 12, 1093-1123.

Kanai, R., van der Geest, J., Frens, M., 2003. Inhibition of saccade initiation by preceding smooth pursuit. Exp. Brain Res. 148, 300-307.

Kerzel, D., Souto, D., Ziegler, N.E., 2008. Effects of attention shifts to stationary objects during steady-state smooth pursuit eye movements. Vision. Res. 48, 958-969.

Khan, A.Z., Lefèvre, P., Heinen, S.J., Blohm, G., 2010. The default allocation of attention is broadly ahead of smooth pursuit. J. Vision. 10 (7-7).

Klimesch, W., 1999. EEG alpha and theta oscillations reflect cognitive and memory performance: a review and analysis. Brain Res. Rev. 29, 169-195.

Kowler, E., 2011. Eye movements: the past 25 years. Vision. Res. 51 (13), 1457-1483.

Kowler, E., Anderson, E., Dosher, B., Blaser, E., 1995. The role of attention in the programming of saccades. Vision. Res. 35, 1897-1916.

Kowler, E., Aitkin, C.D., Ross, N.M., Santos, E.M., Zhao, M., 2014. Davida Teller Award Lecture 2013: the importance of prediction and anticipation in the control of smooth pursuit eye movements. J. Vision. 14 (10-10).

Krauzlis, R.J., 2003. Neuronal activity in the rostral superior colliculus related to the initiation of pursuit and saccadic eye movements. J. Neurosci. 23, 4333-4344.

Krauzlis, R.J., 2004. Recasting the smooth pursuit eye movement system. J. Neurophysiol. 91, 591-603.

Krauzlis, R.J., Dill, N., 2002. Neural correlates of target choice for pursuit and saccades in the primate superior colliculus. Neuron 35, 355-363.

Krauzlis, R.J., Lisberger, S.G., 1994. A model of visually-guided smooth pursuit eye movements based on behavioral observations. J. Comput. Neurosci. 1, 265-283.

Krauzlis, R.J., Lovejoy, L.P., Zénon, A., 2013. Superior colliculus and visual spatial attention. Annu. Rev. Neurosci. 36, 165-182.

Laubrock, J., Engbert, R., Rolfs, M., Kliegl, R., 2007. Microsaccades Are an Index of Covert Attention Commentary on Horowitz, Fine, Fencsik, Yurgenson, and Wolfe (2007). Psychol. Sci. 18, 364-366.

Li, H.-H., Barbot, A., Carrasco, M., 2016. Saccade preparation reshapes sensory tuning 
Curr. Biol. 26, 1564-1570.

Lovejoy, L.P., Fowler, G.A., Krauzlis, R.J., 2009. Spatial allocation of attention during smooth pursuit eye movements. Vision. Res. 49, 1275-1285.

MacLean, G.H., Klein, R.M., Hilchey, M.D., 2015. Does oculomotor readiness mediate exogenous capture of visual attention. J. ExpPsychol Hum. Percept. Perform.

Maioli, C., Falciati, L., Gianesini, T., 2007. Pursuit eye movements involve a covert motor plan for manual tracking. J. Neurosci. 27, 7168-7173.

McMains, S.A., Somers, D.C., 2004. Multiple spotlights of attentional selection in human visual cortex. Neuron 42, 677-686.

McMains, S.A., Somers, D.C., 2005. Processing efficiency of divided spatial attention mechanisms in human visual cortex. J. Neurosci. 25, 9444-9448.

McPeek, R.M., Keller, E.L., 2004. Deficits in saccade target selection after inactivation of superior colliculus. Nat. Neurosci. 7, 757-763.

Moore, T., Armstrong, K.M., 2003. Selective gating of visual signals by microstimulation of frontal cortex. Nature 421, 370-373.

Morgan, S., Hansen, J., Hillyard, S., 1996. Selective attention to stimulus location modulates the steady-state visual evoked potential. Proc. Natl. Acad. Sci. 93, 4770-4774.

Müller, J.R., Philiastides, M.G., Newsome, W.T., 2005. Microstimulation of the superior colliculus focuses attention without moving the eyes. Proc. Natl. Acad. Sci. USA 102, 524-529.

Müller, M.M., Hübner, R., 2002. Can the spotlight of attention be shaped like a doughnut? Evidence from steady-state visual evoked potentials. Psychol. Sci. 13, 119-124.

Müller, M.M., Malinowski, P., Gruber, T., Hillyard, S., 2003. Sustained division of the attentional spotlight. Nature 424, 309-312.

Niehorster, D.C., Siu, W.W., Li, L., 2015. Manual tracking enhances smooth pursuit eye movements. J. Vision. 15 (11-11).

Norcia, A.M., Tyler, C.W., 1985. Spatial frequency sweep VEP: visual acuity during the first year of life. Vision. Res. 25, 1399-1408.

Norcia, A.M., Appelbaum, L.G., Ales, J.M., Cottereau, B.R., Rossion, B., 2015. The steadystate visual evoked potential in vision research: a review. J. Vision. 15 (4-4).

Orban de Xivry, J.J., Lefevre, P., 2007. Saccades and pursuit: two outcomes of a single sensorimotor process. J. Physiol. 584, 11-23.

Pelli, D.G., 1997. The VideoToolbox software for visual psychophysics: Transforming numbers into movies. Spatial Vision 10, 437-442.

Pelli, D.G., 2008. Crowding: a cortical constraint on object recognition. Curr. Opin. Neurobiol. 18, 445-451.

Rizzolatti, G., Riggio, L., Dascola, I., Umiltá, C., 1987. Reorienting attention across the horizontal and vertical meridians: evidence in favor of a premotor theory of attention. Neuropsychologia 25, 31-40.

Rolfs, M., Jonikaitis, D., Deubel, H., Cavanagh, P., 2011. Predictive remapping of attention across eye movements. Nat. Neurosci. 14, 252-256.

Rossion, B., Boremanse, A., 2011. Robust sensitivity to facial identity in the right human occipito-temporal cortex as revealed by steady-state visual-evoked potentials. J. Vision. 11 (16-16).

Schütz, A.C., Braun, D.I., Gegenfurtner, K.R., 2009. Object recognition during foveating eye movements. Vision. Res. 49, 2241-2253.

Schütz, A.C., Braun, D.I., Gegenfurtner, K.R., 2011. Eye movements and perception: a selective review. J. Vision. 11 (9-9).

Seya, Y., Mori, S., 2012. Spatial attention and reaction times during smooth pursuit eye movement. Atten. Percept. Psychophys. 74, 493-509.

Smith, D.T., Schenk, T., 2012. The premotor theory of attention: time to move on? Neuropsychologia 50, 1104-1114.

Spering, M., Schütz, A.C., Braun, D.I., Gegenfurtner, K.R., 2011. Keep your eyes on the ball: smooth pursuit eye movements enhance prediction of visual motion. J. Neurophysiol. 105, 1756-1767.

Störmer, V.S., Alvarez, G.A., Cavanagh, P., 2014. Within-hemifield competition in early visual areas limits the ability to track multiple objects with attention. J. Neurosci. 34, 11526-11533.

Tanaka, M., Yoshida, T., Fukushima, K., 1998. Latency of saccades during smooth-pursuit eye movement in man Directional asymmetries. Exp. Brain Res. 121, 92-98.

Turatto, M., Valsecchi, M., Tame, L., Betta, E., 2007. Microsaccades distinguish between global and local visual processing. Neuroreport 18, 1015-1018.

van Donkelaar, P., 1999. Spatiotemporal modulation of attention during smooth pursuit eye movements. Neuroreport 10, 2523-2526.

van Donkelaar, P., Drew, A.S., 2002. The allocation of attention during smooth pursuit eye movements. Progress. Brain Res. 140, 267-277.

Watamaniuk, S.N., Heinen, S.J., 2015. Allocation of attention during pursuit of large objects is no different than during fixation. J. Vision. 15 (9-9).

Yeshurun, Y., Rashal, E., 2010. Precueing attention to the target location diminishes crowding and reduces the critical distance. J. Vision. 10 (16-16).

Yuval-Greenberg, S., Merriam, E.P., Heeger, D.J., 2014. Spontaneous microsaccades reflect shifts in covert attention. J. Neurosci. 34, 13693-13700.

Zhang, P., Jamison, K., Engel, S., He, B., He, S., 2011. Binocular rivalry requires visual attention. Neuron 71, 362-369. 